\title{
ANÁLISIS PSICOLINGÜÍSTICO DEL DESARROLLO FONÉTICO- FONOLÓGICO DE ALUMNOS PREESCOLARES DE LIMA METROPOLITANA
}

Alejandro S. Dioses Ch. ${ }^{1}$, Lupe Garcia A. ${ }^{2}$, Maria Matalinares C. ${ }^{3}$, Abel Cuzcano Z. ${ }^{4}$, Noemi Panca Ch., Janet Quiroz W., Carla Fernández R., Jenny Castillo R.

(Recibido el 30/10/2006, aceptado el 02/11/2006)

\section{RESUMEN}

Siguiéndose el planteamiento de las reglas fonológicas, se realizó un estudio descriptivo del desarrollo fonético-fonológico de alumnos preescolares de Lima Metropolitana seleccionados mediante un muestreo aleatorio estratificado. La recolección de la información se efectuó con el Test de Desarrollo Fonético-Fonológico conformado por dos áreas: conciencia fonológica y reglas fonológicas, evaluándose fonemas consonánticos y vocálicos de la lengua española.

Los resultados mostraron que existían diferencias significativas en el uso de la conciencia fonológica de rima y aliteración, según el nivel socioeconómico; sin embargo, las diferencias eran no significativas al considerarse las variables de sexo y edad. También se encontraron diferencias no significativas en el uso de las reglas fonológicas en función del sexo, edad y nivel socioeconómico.

Palabras clave: Psicolingüística; Desarrollo Fonético-Fonológico.

\begin{abstract}
Following the approach of the phonological rules, a descriptive study was conducted of the phonetic/phonological development of pre-school students in Metropolitan Lima, who were selected by a stratified random sampling process. Data was collected with the PhoneticPhonological Development Test composed of two areas: phonology awareness and phonology rules, evaluating consonantal and vocalic phonemes of the Spanish language.

The results showed that there were significant differences in the use of phonology awareness of rhyme and alliteration according to the socio-economic level; however, the differences were not significant when considering sex and age variables. No significant differences were also found in the use of phonological rules according to sex, age and socioeconomic level.

Keywords: Psicolinguistic; Phonetic-Phonological Development.

\footnotetext{
Docente Principal, Facultad de Psicología de la UNMSM. E-mail: adiosesch@unmsm.edu.pe

Docente Principal, Facultad de Psicología de la UNMSM. E-mail: Igarciaa@unmsm.edu.pe

3 Docente Asociada, Facultad de Psicología de la UNMSM. E-mail: mmatalinaresc@unmsm.edu.pe

4 Docente Asociado, Facultad de Psicología de la UNMSM. E-mail: mmatalinaresc@unmsm.edu.pe

5 Docente J.P. de la Facultad de Psicologia de la UNMSM. E-mail: npancac@unmsm.edu.pe
} 


\section{INTRODUCCIÓN}

Realizar un estudio del desarrollo fonético-fonológico infantil de nuestra lengua, conlleva efectuar una revisión sucinta de cómo diferentes autores, cada uno desde su posición teórica, ha trabajado este tópico. Así, las investigaciones acerca de la adquisición fonética- fonológica, parten de la propuesta de Jakobson (Acosta; León, Ramos, 1998), quien planteaba la existencia de dos periodos discontinuos y distintivos en su desarrollo: el balbuceo y el habla con significado, esbozando un orden de adquisición innato y universal; sin embargo, esta idea fue descartada porque plantea la inexistencia de una continuidad entre estos periodos surgiendo, en respuesta a ello, la teoría de la fonología natural de Stampe, citado por Bosch (Puyuelo; Rondal, 2003), quien propone un estudio pionero, en el que describe una forma de caracterización de las diferencias entre el habla del niño y la forma de las palabras en la lengua del adulto, centrándose en la noción de procesos fonológicos de simplificación del habla, como mecanismos que le permiten al niño expresarse, aun cuando sus capacidades de habla, le impidan reproducir adecuadamente todos los rasgos y estructuras fonológicas de su lengua. Así también, reporta que la adquisición fonológica es un proceso no acumulativo, debido a que el desarrollo del lenguaje, desde esta perspectiva, representa la pérdida gradual de la intervención de estos procesos innatos y universales que conforman sus experiencias, y que aprenden a suprimir, en razón de que no ocurren en su lenguaje.

Por otro lado, Waterson (Acosta; León; Ramos, 1998: 39) en 1981, propuso un lineamiento de investigación basado en los aspectos perceptivos del lenguaje, centrándose específicamente en las características fonéticas suprasegmentales, tales como la entonación y acento del lenguaje, aunque también consideró las características segmentales. En este sentido, se asume que la percepción y la producción del habla, aún están desarrollándose durante los estadios tempranos del habla con significado, estableciéndose que los niños perciben las producciones del adulto como unidades no analizadas, a partir de las cuales, intentan reproducir sus características más sobresalientes, sin embargo, esta teoría también fue dejada de lado por su falta de alcance en cuanto a la muestra estudiada y al hecho de que su información sólo correspondía a estadios iniciales de adquisición. De otro lado, están los planteamientos de Mowrer (Acosta; León; Ramos, 1998:41), quien explicó el aprendizaje del habla, la imitación y el reforzamiento diferencial, en función a la discriminación de las características propias de los sonidos, enfatizando el papel del refuerzo contingente del adulto en la adquisición fonológica del niño, sin embargo, esta propuesta también fue abandonada, ya que carecía de comprobación empírica sobre el refuerzo como factor principal en la adquisición de los sonidos del habla, y por otorgar al niño, un papel mecánico y automático. Por otro lada parte, Locke, citado por Acosta, León y Ramos (1998, 48), realizó investigaciones en función a las similitudes entre los patrones fonológicos del balbuceo tardío y los del habla temprana con significado, reconociendo tres estadios principales, el prelingüístico, donde el niño es consciente que sus vocalizaciones expresan información con respecto a sus necesidades; el periodo de producción de palabras convencionales; y el referido al habla con significado, no obstante, esta teoría ha sido cuestionada, tanto por el énfasis puesto en los patrones universales del desarrollo, obviando las diferencias individuales tempranas entre sujetos; como por la falta de uso de las estrategias fonológicas en los estudios tempranos, en la que se asigna al niño un papel pasivo.

Ese mismo año, Macken y Ferguson (Acosta, León y Ramos, 1998:47) investigaron las diferencias individuales en el habla de los sujetos, centrando su estudio en los estadios 
tempranos de adquisición fonológica, reconociendo no obstante, la existencia de patrones universales en la adquisición del habla. Esta visión sostenía que los niños formulaban hipótesis sobre el sistema fonológico que estaban adquiriendo, y luego, a través de su experiencia lingüística, comprobaban y revisaban estas hipótesis.

Adicionalmente, es necesario mencionar que todo lo anteriormente descrito, ha llevado al surgimiento de una diversidad de enfoques en el estudio de las dificultades del lenguaje, tratando de explicar los errores de pronunciación del niño en función a problemas motores o perceptivos. Sin embargo, de estas perspectivas de investigación, se ha ido evolucionando a planteamientos de naturaleza cognitiva y lingüística, originando con ello, un cambio sustancial en las estrategias de análisis y tratamiento de las dificultades fonológicas en la población infantil, llegando a proponerse enfoques que enfatizan más bien, en la adquisición por parte del niño, de una serie de reglas que sirven para organizar los elementos fonéticos, que en problemas de tipo físico o fisiológico. Es en esta perspectiva, que la presente investigación cobra sentido, ya que el asumir el punto de vista de la adquisición de reglas fonológicas, estuvo orientada a efectuar un análisis psicolinguístico del desarrollo fonéticofonológico de una población infantil cuyas edades oscilaron entre los 3 y 5 años. Para ello se utilizó el Test de Desarrollo Fonético- Fonológico, conformado por dos áreas: la conciencia fonológica y las reglas fonológicas. En cuanto a la primera área, es pertinente explicar que este constructo hace referencia a una parte del conocimiento metalingüístico, siendo definida por Tunmer y Herriman ( Jiménez; Del Rosario, 2000:23), como la habilidad metalingüística del sujeto para reflexionar y manipular los aspectos estructurales del lenguaje hablado y para tratar el lenguaje como un objeto de conocimiento en sí mismo, siendo conocida también como el metaconocimiento fonológico; así mismo, es importante indicar que esta entidad está conformada por diferentes niveles de conciencia fonológica, en función de la unidad lingüística objeto de reflexión y manipulación por parte del niño, de esta manera, se tiene que este constructo se conforma por una conciencia de la rima y aliteración, primer nivel en la adquisición del conocimiento fonológico, el cual consiste en descubrir que dos palabras comparten un mismo grupo de sonidos; conciencia silábica, entendida como el conocimiento explícito de que las palabras están formadas por una secuencia de unidades fonológicas discretas, cuya característica definitoria, es la de construir unidades articulatorias; conciencia intrasilábica, definida por Arnaiz y Ruiz (2001) como la habilidad para segmentar las sílabas en sus componentes intrasilábicos de principio (onset) y final (rima) ; y conciencia segmentaria, planteada como la habilidad que presta atención consciente a los sonidos de las palabras como unidades abstractas y manipulables (Adams citado por Arnaiz; Ruiz, 2001). Con relación a lo expuesto sobre los niveles de conciencia fonológica, cabe señalar que la presente investigación consideró sólo el nivel de conciencia de rima y aliteración, pues era la que se ajustaba a los propósitos del estudio.

Con respecto al área de las reglas fonológicas, es importante destacar que ellas se encargan de convertir las representaciones internas fonológicas en diferentes manifestaciones fonéticas, es decir, es una operación de sustitución de un fonema por uno de sus alófonos. Contreras y Lleó (Acosta; León; Ramos, 1998) afirman que la propiedad más notable de las reglas fonológicas, es que operan sobre estas clases naturales de sonidos, es decir, sobre sonidos que tienen rasgos fonéticos comunes. Con respecto a esta entidad, el presente estudio planteó el análisis de tres reglas fonológicas; la primera para los fonemas con una sola aparición fonética, en la que se asumía que la aparición de los sonidos no ocurría al mismo tiempo en todas las posiciones, en relación a ello, Templin, citado por Imgran (Acosta; 
León; Moreno, 1998), reportó que la posición inicial es la más facil para las consonantes, seguida por la posición media, y en último lugar la posición final; y las dos restantes, relacionadas con las reglas alofónicas y de neutralización, que, según Dinnsen (Acosta; León; Moreno, 1998), se presentan en el repertorio del sujeto en función al conocimiento que éste posee del sistema fonológico de su comunidad, además de aplicarse también a la descripción de los problemas funcionales del habla y a los distintos grados de conocimiento poseído por los niños con problemas articulatorios. Así, la regla de neutralización define el proceso de adquisición del sistema fonológico del hablante, en el cual las distinciones fonológicas se reducen a un contexto particular (Schane, 1979), entendiéndose por ello que los fonemas pierden su poder de contraste en un contexto concreto. Por otra parte, las reglas alofónicas son las que se encargan de relacionar el inventario fonético (representación superficial) con el fonémico (representación subyacente), siendo su función la conversión de un fonema en uno de sus alófonos; hallando que ésta se da de forma automática, es decir, el alófono se realiza siempre que aparezca en el contexto adecuado.

Complementariamente a lo expuesto, se debe indicar que al ser una investigación efectuada con niños preescolares, fue de suma importancia considerar sus características psicológicas, ya que ellas determinaron, en gran medida, la conformación de los reactivos para la evaluación, entre ellas, el aspecto cognitivo, en el que se destaca como característica principal, el paso de un pensamiento prelógico o preconceptual adquirido durante la primera fase de esta etapa, a uno lógico hacia fines de la misma (que corresponde a la etapa escolar), donde se inician las primeras representaciones mentales, alcanzando más coherencia las acciones y la interacción con la realidad.

Al respecto, Piaget (Papalia; Wendkos, 1998) plantea que esta es la etapa del pensamiento preoperacional, caracterizada porque el niño comienza a utilizar los símbolos y el pensamiento se hace más flexible, de tal manera que la función simbólica se manifiesta a través del lenguaje, la imitación diferida y el juego simbólico. En este periodo, los niños comienzan a entender identidades, funciones y algunos aspectos de clases y relaciones, pero todo se ve limitado por el egocentrismo.

Otro aspecto importante, es el referido a la capacidad perceptiva, en la que el niño incrementa sus posibilidades de discriminación con respecto al propio cuerpo. Así mismo, cabe mencionar que el lenguaje en estos periodos, permite organizar y desarrollar el pensamiento, además de ayudarlo a expresar sentimientos y emociones. Es importante señalar que la adquisición del lenguaje oral, no se da por simple imitación de imágenes y palabras, sino porque el niño ha creado su propia explicación, ha buscado regularidades coherentes, y ha puesto a prueba anticipaciones, creando su propia gramática en forma selectiva de acuerdo a la información que le brinda su medio.

Por último, es necesario señalar que a nivel latinoamericano, no existen trabajos que hayan explorado el desarrollo fonético-fonológico haciendo uso de las reglas fonológicas. Sin embargo, un referente manejado por diversos investigadores, es María Melgar (1976), quien diseño un modelo descriptivo de la articulación, teniendo en cuenta el punto y modo de la misma.

En nuestro país no existen estudios amplios, salvo los realizados por Gonzáles (1990, 1997, 1998), quien analizó los diversos aspectos de la problemática del lenguaje desde su planteamiento psicolinguístico, aunque sin efectuar una caracterización basada en reglas 
fonológicas; y otros en poblaciones puntuales, considerándose aspectos como las habilidades psicolingüísticas (Hugo, 1989), y en los últimos años, las habilidades metalingüísticas (Panca, 2002). Así mismo, se encontró que Caravedo (1998) realizó una serie de estudios fonéticos centrados en el español de Lima, específicamente el relacionado al estudio de la /s/ a partir de una muestra limeña, en la que se analizan los procesos vinculados a la sibilantes: aspiración, interdentalización, sonorización y pérdida de /s/; donde la autora procura demostrar que la variación, supuestamente libre, parece estar regulada por condiciones contextuales, este análisis se realizó en función a los lineamientos dados por el Proyecto Coordinado del Habla de las Principales Ciudades Hispanoamericanas, dirigido por Lope Blanch (Caravedo, 1998). Con relación a los resultados de su estudio, la autora encontró una jerarquía contextual asociada a las manifestaciones de /s/, la cual regula la coexistencia de variables en el grupo social investigado, así mismo encontró una significativa relación inversa de ese orden jerárquico, entre los procesos de mantenimiento, por un lado, y de aspiración y pérdida, por otro; hallándose además, que las correlaciones de sexo y generación se muestran poco significativas en cuanto no alteran el orden de los contextos, sino que lo reafirman.

Por ello, en razón a todo lo señalado, se estimó pertinente plantear las siguientes hipótesis:

H1: Los preescolares que asisten a centros de educación inicial de Lima Metropolitana muestran diferencias significativas en conciencia fonológica de rima y aliteración, en razón a la edad, sexo y nivel socioeconómico al que pertenecen.

H2: Los preescolares que asisten a centros de educación inicial de Lima Metropolitana muestran diferencias significativas en las reglas fonológicas en razón a la edad, sexo y nivel socioeconómico al que pertenecen.

\section{MÉTODO}

Método: El método utilizado en la presente investigación fue el descriptivo (Ugarriza, 2001:28) con un diseño transversal-comparativo (Hernández, 1999). Las variables independientes fueron la edad, sexo y nivel socioeconómico; mientras que la variable dependiente estuvo dada por el desarrollo fonético fonológico.

Sujetos: La muestra del estudio estuvo conformada por 601 alumnos (Tabla 1) del nivel inicial jardín, pertenecientes a instituciones educativas públicas y privadas de Lima Metropolitana de diferente segmento socioeconómico (A, B, C, D y E), cuyas edades fluctuaron entre los 3 y 5 años, seleccionados mediante un muestreo aleatorio estratificado y distribuidos de la siguiente manera: 
Tabla 1. Distribución de la muestra por nivel socioeconómico, edad y sexo.

\begin{tabular}{|c|c|c|c|c|c|c|c|c|}
\hline & \multirow[b]{3}{*}{ Edad } & \multicolumn{6}{|c|}{ Sexo } & \multirow{3}{*}{ Total } \\
\hline & & \multicolumn{3}{|c|}{ Femenino } & \multicolumn{3}{|c|}{ Masculino } & \\
\hline & & 3 años & 4 años & 5 años & 3 años & 4 años & 5 años & \\
\hline \multirow{5}{*}{ 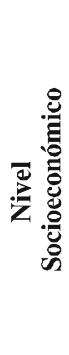 } & $\mathbf{A}$ & 5 & 8 & 7 & 4 & 10 & 5 & 39 \\
\hline & B & 13 & 14 & 17 & 16 & 17 & 10 & 87 \\
\hline & C & 35 & 23 & 28 & 24 & 32 & 27 & 169 \\
\hline & D & 21 & 31 & 33 & 33 & 30 & 30 & 178 \\
\hline & $\mathbf{E}$ & 21 & 22 & 22 & 21 & 22 & 20 & 128 \\
\hline \multirow{2}{*}{\multicolumn{2}{|c|}{ Total }} & 95 & 98 & 107 & 98 & 111 & 92 & \multirow{2}{*}{601} \\
\hline & & \multicolumn{3}{|c|}{300} & \multicolumn{3}{|c|}{301} & \\
\hline
\end{tabular}

Instrumento: La recolección de los datos se efectuó utilizando el Test de Desarrollo Fonético-Fonológico (TDFF), tal como se detalla a continuación:

Denominación: Test de Desarrollo Fonético-Fonológico

Autor: Alejandro S. Dioses Chocano

Colaboradores: $\quad$ S. Manrique; J. Castillo; C. Fernández; J. Quiroz

Año: 2004

Procedencia: $\quad$ Perú

Institución: $\quad$ CPAL-Centro de Investigación y Publicaciones

Administración: Individual

Duración:

20 minutos aproximadamente

Aplicación: Niños y niñas de 3, 4 y 5 años de edad

Significación: $\quad$ Valoración del grado de desarrollo fonético-fonológico en niños y niñas de 3 a 5 años de edad.

El test está distribuido en dos áreas, una relacionada a la evaluación de la conciencia fonológica de rima y aliteración conformada por un total de 17 tarjetas, cada una de las cuales evalúa un fonema consonántico determinado; la otra configurada en tres subáreas: reglas con fonemas de una sola aparición fonética, reglas alofónicas y reglas de neutralización. La evaluación de esta área se efectuó simultáneamente mediante la narración de tres cuentos, de cinco secuencias cada uno.

El manual informa que la confiabilidad del instrumento se obtuvo mediante la técnica de consistencia interna, entendida como la intercorrelación entre los ítemes, utilizándose para ello, el coeficiente Alfa de Crombach. 
La validez fue juzgada a través del contenido, para lo cual se determinó que la muestra de reactivos era representativa del universo de ítems referentes al desarrollo fonológico, siendo los jueces, tres psicólogos y tres profesores con más de diez años de experiencia de trabajo con estos niveles de edad y tipos de contenidos.

\section{RESULTADOS}

El tratamiento de los resultados se efectuó mediante el paquete estadístico SPSS versión 11 en español. El análisis descriptivo del desempeño en conciencia fonológica de rima y aliteración considerándose la edad (Tabla 2), mostró que la media alcanzada por los alumnos se incrementó conforme avanzó la edad. Cabe resaltar que si bien el aumento es progresivo, éste es mínimo.

Tabla 2. Puntaje total de la media de conciencia fonológica de rima y aliteración según la edad.

\begin{tabular}{lccccccc}
\cline { 2 - 7 } & \multicolumn{4}{c}{3 años } & \multicolumn{2}{c}{4 años } & \multicolumn{2}{c}{5 años } \\
\cline { 2 - 8 } & Media & DS & Media & DS & Media & DS \\
\hline Conciencia fonológica & 15,23 & 1,969 & 16,08 & 1,349 & 16,28 & 1,083 \\
\hline
\end{tabular}

El análisis descriptivo del desempeño en conciencia fonológica de rima y aliteración considerándose la variable sexo (Tabla 3), mostró que el desarrollo de los niños fue ligeramente superior al alcanzado por las niñas.

Tabla 3. Puntaje total de la media de conciencia fonológica de rima y aliteración según el sexo.

\begin{tabular}{lcccc}
\cline { 2 - 5 } & \multicolumn{4}{c}{ Sexo } \\
\cline { 2 - 5 } & \multicolumn{2}{c}{ Femenino } & \multicolumn{2}{c}{ Masculino } \\
Media & DS & Media & DS \\
\hline Conciencia fonológica & 15,86 & 1,570 & 15,88 & 1,569 \\
\hline
\end{tabular}

Luego, al considerarse la variable nivel socioeconómico en la descripción del desempeño logrado por los alumnos en conciencia fonológica de rima y aliteración (Tabla 4), se apreció que la media aumentó de manera progresiva conforme el nivel socioeconómico del alumno fue más alto. 
Tabla 4. Puntaje total de la media de conciencia fonológica de rima y aliteración según el nivel socioeconómico.

\begin{tabular}{|c|c|c|c|c|c|c|c|c|c|c|}
\hline & \multicolumn{10}{|c|}{ Nivel socioeconómico } \\
\hline & \multicolumn{2}{|c|}{ A } & \multicolumn{2}{|c|}{ B } & \multicolumn{2}{|c|}{$\mathrm{C}$} & \multicolumn{2}{|c|}{$\mathrm{D}$} & \multicolumn{2}{|c|}{ E } \\
\hline & Media & DS & Media & DS & Media & DS & Media & DS & Media & DS \\
\hline $\begin{array}{l}\text { Conciencia } \\
\text { fonológica }\end{array}$ & 16,64 & 0,584 & 16,18 & 1,410 & 15,92 & 1,486 & 15,80 & 1,559 & 15,47 & 1,857 \\
\hline
\end{tabular}

Con respecto al rendimiento alcanzado por los alumnos en las evaluaciones de reglas alofónicas (Tabla 5), se observó un mejor desempeño conforme aumentó la edad; es así que la puntuación media de los alumnos de 5 años $(44,84)$ fue mayor que la puntuación media de los alumnos de 4 años $(44,35)$ y de 3 años $(44,26)$.

Tabla 5. Puntaje total de la media de reglas alofónicas según la edad.

\begin{tabular}{ccc}
\hline Edad & Media & DS \\
\hline 3 años & 44,26 & 3,891 \\
4 años & 44,35 & 3,670 \\
5 años & 44,84 & 3,217 \\
\hline Total & 44,49 & 3,605 \\
\hline
\end{tabular}

El análisis descriptivo del desempeño en reglas alofónicas, considerándose la variable sexo (Tabla 6), mostró que la puntuación promedio de las niñas $(44,81)$ fue ligeramente superior a la alcanzada por los niños $(44,16)$, siendo la desviación estándar de las niñas menor que la de los niños.

Tabla 6. Puntaje total de la media de las reglas alofónicas según el sexo.

\begin{tabular}{ccc}
\hline Sexo & Media & DS \\
\hline Masculino & 44,16 & 4,212 \\
Femenino & 44,81 & 2,839 \\
\hline
\end{tabular}

Por otro lado, al considerarse la variable nivel socioeconómico, se observó que el desempeño logrado por los alumnos en reglas alofónicas (Tabla 7) no mostró un patrón de incremento progresivo de la media conforme fue más alto el nivel socioeconómico, sino más bien se observó un rendimiento irregular, es así que los alumnos de NSE A $(46,90)$ evidenciaron un rendimiento mayor con respecto a los alumnos de NSE B $(44,98)$; mientras que los niños de NSE C $(47,08)$ y NSE D $(47,10)$ mostraron un mejor desempeño con relación a los anteriores. 
Sin embargo, los alumnos de NSE E $(46,52)$ tuvieron un desempeño más bajo con respecto a sus compañeros de NSE A, NSE C y NSE D; pero un desempeño mejor con relación a sus compañeros de NSE B.

Tabla 7. Puntaje total de la media de las reglas alofónicas según el nivel socioeconómico.

\begin{tabular}{lcc}
\hline $\begin{array}{l}\text { Nivel } \\
\text { socieconómico }\end{array}$ & Media & DS \\
\hline NSE A & 46,90 & 6,125 \\
NSE B & 44,98 & 7,294 \\
NSE C & 47,08 & 4,698 \\
NSE D & 47,10 & 4,304 \\
NSE E & 46,52 & 4,196 \\
\hline
\end{tabular}

Con relación a la media alcanzada por los alumnos en las evaluaciones de reglas de neutralización (Tabla 8), se observó un mejor desempeño conforme aumentó la edad; es así que la puntuación media de los alumnos de 5 años $(7,36)$ fue mayor que la puntuación media de los alumnos de 4 años $(7,02)$ y de 3 años $(6,30)$.

Tabla 8. Puntaje total de la media de las reglas de neutralización según la edad.

\begin{tabular}{lcc}
\hline Edad & Media & DS \\
\hline 3 años & 6,30 & 2,580 \\
4 años & 7,02 & 2,322 \\
5 años & 7,36 & 2,357 \\
\hline
\end{tabular}

Por otra parte el análisis descriptivo del desempeño en reglas de neutralización, considerándose la variable sexo (Tabla 9), mostró que el desarrollo de las niñas es superior al alcanzado por los niños.

Tabla 9. Puntaje total de la media de las reglas de neutralización según el sexo.

\begin{tabular}{clc}
\hline Sexo & Media & DS \\
\hline Masculino & 6,65 & 2,457 \\
Femenino & 7,15 & 2,431 \\
\hline
\end{tabular}

Con respecto al desempeño logrado por los alumnos en las reglas de neutralización (Tabla 10), teniendo en cuenta la variable nivel socioeconómico, se apreció que la media de los alumnos de NSE A $(8,13)$ evidenció un rendimiento mayor con respecto a los alumnos de 
NSE B $(6,57)$; mientras que los niños de NSE C $(6,91)$ presentaron un mejor rendimiento con respecto a estos últimos; por otro lado, los niños del NSE D $(7,81)$ mostraron un mejor desempeño con relación a los niños de $\operatorname{NSE~E~}(5,48)$, siendo éstos, los alumnos que presentaron el rendimiento más bajo con relación a la variable estudiada.

Tabla 10. Puntaje total de la media de las reglas de neutralización según el nivel socioeconómico.

\begin{tabular}{lll}
\hline $\begin{array}{l}\text { Nivel } \\
\text { socieconómico }\end{array}$ & Media & DS \\
\hline NSE A & 8,13 & 1,704 \\
NSE B & 6,57 & 2,802 \\
NSE C & 6,91 & 2,284 \\
NSE D & 7,81 & 1,971 \\
NSE E & 5,48 & 2,497 \\
\hline
\end{tabular}

En cuanto a la media alcanzada por los alumnos en las evaluaciones de reglas fonológicas (Tabla 11), se observó un mejor desempeño conforme se incrementó la edad; es así que la puntuación media de los alumnos de 5 años $(99,10)$ es mayor que la puntuación media de los alumnos de 4 años $(98,37)$ y de 3 años $(96,58)$. Por otro lado, cabe resaltar que la puntuación media total fue de 98,03 con una desviación estándar de 9,208.

Tabla 11. Puntaje total de la media de las reglas fonológicas según la edad.

\begin{tabular}{cccc}
\hline Edad & Media & N & DS \\
\hline 3 años & 96,58 & 193 & 10,155 \\
4 años & 98,37 & 209 & 8,707 \\
5 años & 99,10 & 199 & 8,602 \\
\hline Total & 98,03 & 601 & 9,208 \\
\hline
\end{tabular}

Así mismo, el análisis descriptivo del desempeño en reglas fonológicas, considerándose la variable sexo (Tabla 12), indicó que el desarrollo de las niñas fue superior al alcanzado por los niños; mientras que la puntuación total fue de 98,03 con una desviación estándar de 9,208 .

Tabla 12. Puntaje total de la media de las reglas fonológicas según el sexo.

\begin{tabular}{rccc}
\hline Sexo & Media & N & DS \\
\hline Masculino & 97,14 & 301 & 9,768 \\
Femenino & 98,94 & 300 & 8,532 \\
\hline Total & 98,03 & 601 & 9,208 \\
\hline
\end{tabular}


Complementariamente, se apreció que el desempeño logrado por los alumnos en las reglas fonológicas (Tabla 13), considerando la variable nivel socioeconómico, evidenció puntajes irregulares caracterizados por altibajos, es así que los alumnos de NSE A (98.54) tuvieron un rendimiento mayor con respecto a los alumnos de NSE B (94.62); mientras que los niños de NSE C (98.72) presentaron un mejor rendimiento con respecto de estos últimos; por otro lado, los niños del NSE D (99.74) tuvieron un mejor desempeño con relación a todos los demás niños. Finalmente, los niños de NSE E (96.92) presentaron un desempeño inferior con respecto a sus compañeros de NSE A, NSE B Y NSE C.

Tabla 13. Puntaje total de la media de las reglas fonológicas según el nivel socioeconómico.

\begin{tabular}{cccc}
\hline Nivel socioeconómico & Media & N & DS \\
\hline NSE A & 98,54 & 39 & 11,914 \\
NSE B & 94,62 & 87 & 14,394 \\
NSE C & 98,72 & 169 & 8,024 \\
NSE D & 99,74 & 178 & 7,013 \\
NSE E & 96,92 & 128 & 7,078 \\
\hline Total & 98,03 & 601 & 9,208 \\
\hline
\end{tabular}

Posteriormente el tratamiento estadístico de las hipótesis planteadas, permitió apreciar que al compararse el desarrollo de la conciencia fonológica de rima y aliteración en función a la variable sexo, no existieron diferencias significativas entre varones y mujeres.

Tabla 14. Comparación del desarrollo de la conciencia fonológica de rima y aliteración en función a la variable sexo.

\begin{tabular}{llcc}
\hline & & Sig. & t de student \\
\hline $\begin{array}{l}\text { Total conciencia fonológica } \\
\text { de rima y aliteración- sexo }\end{array}$ & Varianzas iguales &, 971 & $\mathbf{, 1 5 9}$ \\
& Varianzas diferentes & &, 159 \\
\hline
\end{tabular}

Significación 0,971 > 0,05 se asume el valor $\mathrm{t}_{\mathrm{c}}$ para varianzas iguales.

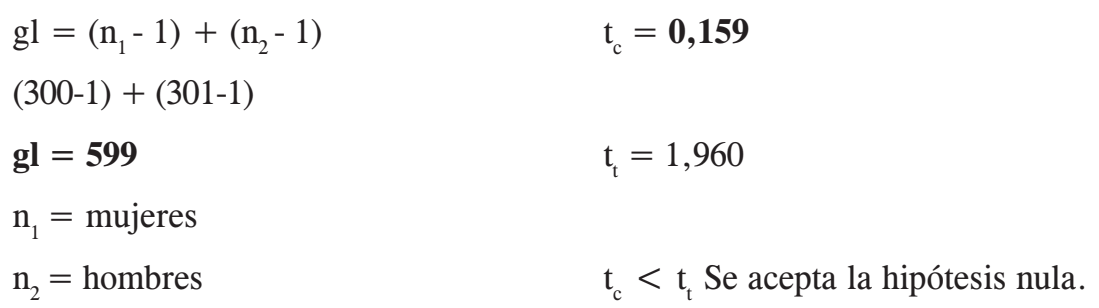


En cuanto a la comparación del desarrollo de la conciencia fonológica de rima y aliteración en función a la variable edad, se apreció que, tanto a nivel de niños de 3 años con respecto a los de 4 años (Tabla 15), como entre los de 3 con respecto a los de 5 años (Tabla 16), no existieron ten diferencias significativas.

Tabla 15. Comparación del desarrollo de la conciencia fonológica de rima y aliteración entre los niños de 3 y 4 años.

\begin{tabular}{llcc}
\hline & & Sig. & t de student \\
\hline $\begin{array}{l}\text { Total conciencia fonológica } \\
\text { de rima y aliteración- edad }\end{array}$ & Varianzas iguales &, 000 & $-5,041$ \\
3 años-4 años & Varianzas diferentes & & $-4,969$ \\
\hline
\end{tabular}

Significación $0,000<0,05$ se asume el valor $\mathrm{t}_{\mathrm{c}}$ para varianzas diferentes.

$$
\begin{array}{ll}
\mathrm{gl}=\left(\mathrm{n}_{1}-1\right)+\left(\mathrm{n}_{2}-1\right) & \mathrm{t}_{\mathrm{c}}=\mathbf{- 4 , 9 6 9} \\
(193-1)+(209-1) & \\
\mathbf{g l}=\mathbf{4 0 0} & \mathrm{t}_{\mathrm{t}}=1,960 \\
\mathrm{n}_{1}=3 \text { años } & \\
\mathrm{n}_{2}=4 \text { años } & \mathrm{t}_{\mathrm{c}}<\mathrm{t}_{\mathrm{t}} \text { Se acepta la hipótesis nula. }
\end{array}
$$

Tabla 16. Comparación del desarrollo de la conciencia fonológica de rima y aliteración entre los niños de 3 y 5 años.

\begin{tabular}{llcc}
\hline & & Sig. & t de student \\
\hline $\begin{array}{l}\text { Total conciencia fonológica } \\
\text { de rima y aliteración- edad }\end{array}$ & Varianzas iguales &, 000 & $-6,556$ \\
3 años - 5 años & Varianzas diferentes & & $-6,503$ \\
\hline
\end{tabular}

Significación ,000 < 0,05 se asume el valor $t_{c}$ para varianzas diferentes.

$$
\begin{array}{ll}
\mathrm{gl}=\left(\mathrm{n}_{1}-1\right)+\left(\mathrm{n}_{2}-1\right) & \mathrm{t}_{\mathrm{c}}=\mathbf{- 6 , 5 0 3} \\
(193-1)+(209-1) & \\
\mathbf{g l}=\mathbf{4 0 0} & \mathrm{t}_{\mathrm{t}}=1,960 \\
\mathrm{n}_{1}=3 \text { años } & \\
\mathrm{n}_{2}=5 \text { años } & \mathrm{t}_{\mathrm{c}}<\mathrm{t}_{\mathrm{t}} \text { Se acepta la hipótesis nula. }
\end{array}
$$


Con respecto a la comparación del desarrollo de la conciencia fonológica de rima y aliteración, en función al nivel socioeconómico, los resultados permitieron apreciar la existencia de diferencias significativas (Tablas 17, 18, 19, 20).

Tabla 17. Comparación del desarrollo de la conciencia fonológica de rima y aliteración entre los segmentos socioeconómicos A y B.

\begin{tabular}{llcc}
\hline & & Sig. & t de student \\
\hline $\begin{array}{l}\text { Total conciencia fonológica } \\
\text { de rima y aliteración-nivel } \\
\text { socioeconómico A - B }\end{array}$ & Varianzas iguales &, 001 & $-1,947$ \\
\hline
\end{tabular}

Significación $0,001<0,05$ se asume el valor $\mathrm{t}_{\mathrm{c}}$ para varianzas diferentes.

$$
\begin{array}{ll}
\mathrm{gl}=\left(\mathrm{n}_{1}-1\right)+\left(\mathrm{n}_{2}-1\right) & \mathrm{t}_{\mathrm{c}}=\mathbf{2 , 5 7 1} \\
(39-1)+(87-1) & \\
\mathbf{g l}=\mathbf{1 2 4} & \mathrm{t}_{\mathrm{t}}=1,980 \\
\mathrm{n}_{1}=\mathrm{A} & \\
\mathrm{n}_{2}=\mathrm{B} & \mathrm{t}_{\mathrm{c}}>\mathrm{t}_{\mathrm{t}} \text { Se rechaza la hipótesis nula. }
\end{array}
$$

Tabla 18. Comparación del desarrollo de la conciencia fonológica de rima y aliteración entre los segmentos socioeconómicos A y C.

\begin{tabular}{llcc}
\hline & & Sig. & t de student \\
\hline $\begin{array}{l}\text { Total conciencia fonológica } \\
\text { de rima y aliteración-nivel } \\
\text { socioeconómico A - C }\end{array}$ & Varianzas iguales &, 004 & 2,985 \\
\hline
\end{tabular}

Significación ,004 $<0,05$ se asume el valor $\mathrm{t}_{\mathrm{c}}$ para varianzas diferentes.

$$
\begin{array}{ll}
\mathrm{gl}=\left(\mathrm{n}_{1}-1\right)+\left(\mathrm{n}_{2}-1\right) & \mathrm{t}_{\mathrm{c}}=\mathbf{4 , 9 0 1} \\
(39-1)+(87-1) & \\
\mathbf{g l}=\mathbf{1 2 4} & \mathrm{t}_{\mathrm{t}}=1,960 \\
\mathrm{n}_{1}=\mathrm{A} & \\
\mathrm{n}_{2}=\mathrm{C} & \mathrm{t}_{\mathrm{c}}>\mathrm{t}_{\mathrm{t}} \text { Se rechaza la hipótesis nula. }
\end{array}
$$


Tabla 19. Comparación del desarrollo de la conciencia fonológica de rima y aliteración entre los segmentos socioeconómicos A-D.

\begin{tabular}{llcc}
\hline & & Sig. & t de student \\
\hline $\begin{array}{l}\text { Total conciencia fonológica } \\
\text { de rima y aliteración-nivel } \\
\text { socioeconómico A - D }\end{array}$ & Varianzas iguales &, 000 & 3,300 \\
\hline
\end{tabular}

Significación $0,000<0,05$ se asume el valor $\mathrm{t}_{\mathrm{c}}$ para varianzas diferentes.

$$
\begin{array}{ll}
\mathrm{gl}=\left(\mathrm{n}_{1}-1\right)+\left(\mathrm{n}_{2}-1\right) & \mathbf{t}_{\mathbf{c}}=\mathbf{5 , 5 9 6} \\
(39-1)+(178-1) & \\
\mathbf{g l}=\mathbf{2 1 5} & \mathrm{t}_{\mathrm{t}}=1,960 \\
\mathrm{n}_{1}=\mathrm{A} & \\
\mathrm{n}_{2}=\mathrm{D} & \mathrm{t}_{\mathrm{c}}>\mathrm{t}_{\mathrm{t}} \text { Se rechaza la hipótesis nula. }
\end{array}
$$

Tabla 20. Comparación del desarrollo de la conciencia fonológica de rima y aliteración entre los segmentos socioeconómicos A y E.

\begin{tabular}{llcc}
\hline & & Sig. & t de student \\
\hline $\begin{array}{l}\text { Total conciencia fonológica } \\
\text { de rima y aliteración - nivel } \\
\text { socioeconómico A - E }\end{array}$ & Varianzas iguales &, 000 & 3,877 \\
\hline
\end{tabular}

Significación $.000<0.05$ se asume el valor $\mathrm{t}_{\mathrm{c}}$ para varianzas diferentes.

$$
\begin{array}{ll}
\mathrm{gl}=\left(\mathrm{n}_{1}-1\right)+\left(\mathrm{n}_{2}-1\right) & \mathrm{t}_{\mathrm{c}}=\mathbf{6 , 2 0 5} \\
(39-1)+(128-1) & \\
\mathrm{gl}=\mathbf{1 6 5} & \mathrm{t}_{\mathrm{t}}=1,960 \\
\mathrm{n}_{1}=\mathrm{A} & \\
\mathrm{n}_{2}=\mathrm{E} & \mathrm{t}_{\mathrm{c}}>\mathrm{t}_{\mathrm{t}} \text { Se rechaza la hipótesis nula. }
\end{array}
$$

En cuanto a la comparación del desarrollo de las reglas fonológicas en función a la variable sexo, se apreció (Tabla 21), que no existieron diferencias significativas entre niños y niñas. 
Tabla 21. Comparación del desarrollo de las reglas fonológicas en función a la variable sexo.

\begin{tabular}{llcc}
\hline & & Sig. & t de student \\
\hline Total reglas fonológicas - & Varianzas iguales &, 350 & $-2,406$ \\
sexo & Varianzas diferentes & & $-2,407$ \\
\hline
\end{tabular}

Significación .350 $>0.05$ se asume el valor $\mathrm{t}_{\mathrm{c}}$ para varianzas iguales.

$$
\begin{array}{ll}
\mathrm{gl}=\left(\mathrm{n}_{1}-1\right)+\left(\mathrm{n}_{2}-1\right) & \mathrm{t}_{\mathrm{c}}=\mathbf{- 2 , 4 0 6} \\
(300-)+(301-) & \\
\mathrm{gl}=599 & \mathrm{t}_{\mathrm{t}}=1,960 \\
\mathrm{n}_{1}=\text { mujeres } & \\
\mathrm{n}_{2}=\text { hombres } & \mathrm{t}_{\mathrm{c}}<\mathrm{t}_{\mathrm{t}} \text { Se acepta la hipótesis nula. }
\end{array}
$$

En la comparación del desarrollo de las reglas fonológicas entre los niños de 3 y 4 años así como entre los de 3 y 5 años, tal como lo muestran las Tablas 22 y 23, no existieron diferencias significativas.

Tabla 22. Comparación del desarrollo de las reglas fonológicas entre los niños de 3 y 4 años.

\begin{tabular}{llcc}
\hline & & Sig. & t de student \\
\hline Total reglas fonológicas - edad & Varianzas iguales &, 74 & $-1,899$ \\
3 años - 4 años & Varianzas diferentes & & $-1,888$ \\
\hline
\end{tabular}

Significación 0,74 >0,05 se asume el valor $\mathrm{t}_{\mathrm{c}}$ para varianzas iguales.

$$
\begin{array}{ll}
\mathrm{gl}=\left(\mathrm{n}_{1}-1\right)+\left(\mathrm{n}_{2}-1\right) & \mathrm{t}_{\mathrm{c}}=\mathbf{- 1 , 8 9 9} \\
(193-1)+(209-1) & \\
\mathrm{gl}=400 & \mathrm{t}_{\mathrm{t}}=1,960 \\
\mathrm{n}_{1}=3 \text { años } & \\
\mathrm{n}_{2}=4 \text { años } & \mathrm{t}_{\mathrm{c}}<\mathrm{t}_{\mathrm{t}} \text { Se acepta la hipótesis nula. }
\end{array}
$$


Tabla 23. Comparación del desarrollo de las reglas fonológicas entre los niños de 3 y 5 años.

\begin{tabular}{llcc}
\hline & & Sig. & t de student \\
\hline Total reglas fonológicas - edad & Varianzas iguales &, 726 & $\mathbf{- 2 , 6 4 9}$ \\
3 años - 5 años & Varianzas diferentes & & $-2,642$ \\
\hline
\end{tabular}

Significación $0,726>0,05$ se asume el valor $\mathrm{t}_{\mathrm{c}}$ para varianzas iguales.

$$
\begin{array}{ll}
\mathrm{gl}=\left(\mathrm{n}_{1}-1\right)+\left(\mathrm{n}_{2}-1\right) & \mathrm{t}_{\mathrm{c}}=\mathbf{- 2 , 6 4 9} \\
(193-1)+(199-1) & \\
\mathrm{gl}=290 & \mathrm{t}_{\mathrm{t}}=1,960 \\
\mathrm{n}_{1}=3 \text { años } & \\
\mathrm{n}_{2}=5 \text { años } & \mathrm{t}_{\mathrm{c}}>\mathrm{t}_{\mathrm{t}} \text { Se acepta la hipótesis nula. }
\end{array}
$$

Los resultados de la comparación de reglas fonológicas por nivel socioeconómico, indicaron, en todos los casos (Tabla 24, 25, 26, 27), que no existieron diferencias significativas entre los segmentos socioeconómicos mencionados.

Tabla 24. Comparación del desarrollo de las reglas fonológicas entre los segmentos socioeconómicos A y B.

\begin{tabular}{llcc}
\hline & & Sig. & t de student \\
\hline & Varianzas iguales &, 995 & $\mathbf{1 , 4 8 6}$ \\
$\begin{array}{l}\text { Total reglas fonológicas - nivel } \\
\text { socioeconómico A - B }\end{array}$ & Varianzas diferentes & & 1,597 \\
\hline
\end{tabular}

Significación 0,995 > 0,05 se asume el valor $\mathrm{t}_{\mathrm{c}}$ para varianzas iguales.

$$
\begin{array}{ll}
\mathrm{gl}=\left(\mathrm{n}_{1}-1\right)+\left(\mathrm{n}_{2}-1\right) & \mathrm{t}_{\mathrm{c}}=\mathbf{1 , 4 8 6} \\
(39-1)+(87-1) & \\
\mathrm{gl}=124 & \mathrm{t}_{\mathrm{t}}=1,980 \\
\mathrm{n}_{1}=\mathrm{A} & \\
\mathrm{n}_{2}=\mathrm{B} & \mathrm{t}_{\mathrm{c}}<\mathrm{t}_{\mathrm{t}} \text { Se acepta la hipótesis nula. }
\end{array}
$$


Tabla 25. Comparación del desarrollo de las reglas fonológicas entre los segmentos socioeconómicos A y C.

\begin{tabular}{llcc}
\hline & & Sig. & t de student \\
\hline & Varianzas iguales &, 000 &,- 116 \\
$\begin{array}{l}\text { Total reglas fonológicas - nivel } \\
\text { socioeconómico A - C }\end{array}$ & Varianzas diferentes & &,- 091 \\
\hline
\end{tabular}

Significación $0.000<0,05$ se asume el valor $\mathrm{t}_{\mathrm{c}}$ para varianzas diferentes.

$\mathrm{gl}=\left(\mathrm{n}_{1}-1\right)+\left(\mathrm{n}_{2}-1\right) \quad \mathrm{t}_{\mathrm{c}}=-, 091$

$(39-1)+(169-1)$

$\mathrm{gl}=300$

$t_{t}=1,960$

$\mathrm{n}_{1}=\mathrm{A}$

$\mathrm{n}_{2}=\mathrm{C}$

$\mathrm{t}_{\mathrm{c}}<\mathrm{t}_{\mathrm{t}}$ Se acepta la hipótesis nula.

Tabla 26. Comparación del desarrollo de las reglas fonológicas entre los segmentos socioeconómicos A y D.

\begin{tabular}{llcc}
\hline & & Sig. & t de student \\
\hline & Varianzas iguales &, 000 &,- 840 \\
$\begin{array}{l}\text { Total reglas fonológicas - nivel } \\
\text { socioeconómico A - D }\end{array}$ & Varianzas diferentes & & $\mathbf{- , 6 0 8}$ \\
\hline
\end{tabular}

Significación $0.000<0.05$ se asume el valor $\mathrm{t}_{\mathrm{c}}$ para varianzas diferentes.

$\mathrm{gl}=\left(\mathrm{n}_{1}-1\right)+\left(\mathrm{n}_{2}-1\right) \quad \mathrm{t}_{\mathrm{c}}=-.608$

$(39-1)+(178-1)$

$\mathrm{gl}=215$

$\mathrm{t}_{\mathrm{t}}=1.960$

$\mathrm{n}_{1}=\mathrm{A}$

$\mathrm{n}_{2}=\mathrm{D} \quad \mathrm{t}_{\mathrm{c}}<\mathrm{t}_{\mathrm{t}}$ Se acepta la hipótesis nula.

Tabla 27. Comparación del desarrollo de las reglas fonológicas entre los segmentos socioeconómicos A y E.

\begin{tabular}{llcc}
\hline & & Sig. & t de student \\
\hline & Varianzas iguales &, 000 & 1,047 \\
$\begin{array}{l}\text { Total reglas fonológicas - nivel } \\
\text { socioeconómico A - E }\end{array}$ & Varianzas diferentes & & $\mathbf{0 , 8 0 5}$ \\
\hline
\end{tabular}


Significación $0,000<0,05$ se asume el valor $\mathrm{t}_{\mathrm{c}}$ para varianzas diferentes.

$$
\begin{array}{ll}
\mathrm{gl}=\left(\mathrm{n}_{1}-1\right)+\left(\mathrm{n}_{2}-1\right) & \mathrm{t}_{\mathrm{c}}=\mathbf{0 , 8 0 5} \\
(39-1)+(128-1) & \\
\mathrm{gl}=165 & \mathrm{t}_{\mathrm{t}}=1,960 \\
\mathrm{n}_{1}=\mathrm{A} & \\
\mathrm{n}_{2}=\mathrm{E} & \mathrm{t}_{\mathrm{c}}<\mathrm{t}_{\mathrm{t}} \text { Se acepta la hipótesis nula. }
\end{array}
$$

Los resultados con respecto al perfil de desarrollo de las reglas fonológicas (Tabla 28), mostraron que éste se incrementó de manera progresiva con relación a la edad cronológica.

Tabla 28. Perfil de desarrollo de las reglas fonológicas.

\begin{tabular}{cccc}
\cline { 2 - 4 } & \multicolumn{3}{c}{ Edad } \\
\cline { 2 - 4 } & 3 años & 4 años & 5 años \\
\hline Media & 96,58 & 98,37 & 99,10 \\
\hline
\end{tabular}

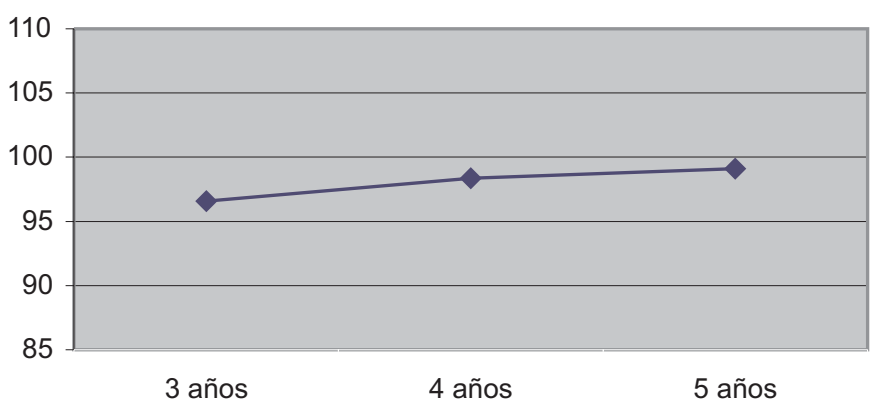

Por ora parte, es estudio de los datos con respecto al desarrollo de la evaluación de conciencia fonológica de rima y aliteración (Tabla 29), mostró que el perfil de desempeño se incrementó de manera progresiva en función a la edad cronológica. 
Tabla 29. Perfil de desarrollo de las reglas fonológicas.

\begin{tabular}{cccc}
\cline { 2 - 4 } & \multicolumn{3}{c}{ Edad } \\
\cline { 2 - 4 } & 3 años & 4 años & 5 años \\
\hline Media & 15,23 & 16,08 & 16,28
\end{tabular}

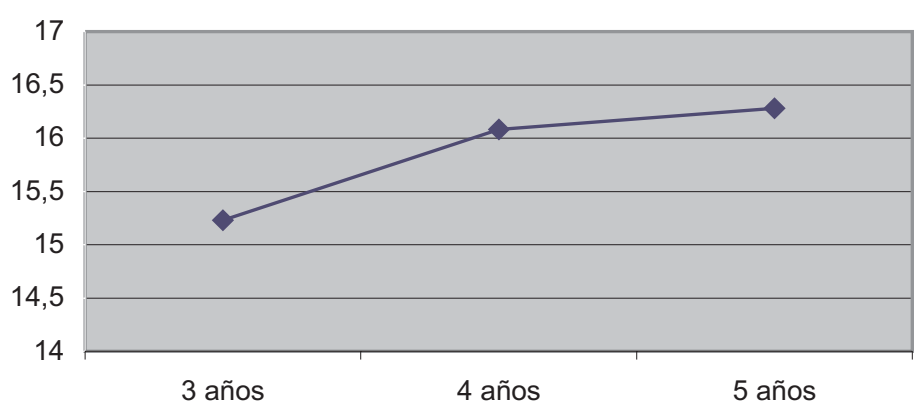

Complementariamente, los resultados, en cuanto al desarrollo de la evaluación de reglas para fonemas con un solo contexto de aparición (Tabla 30), mostraron que el perfil se fue incrementando de manera progresiva conforme fue mayor la edad cronológica.

Tabla 30. Perfil del desarrollo de las reglas para fonemas con un solo contexto de aparición.

\begin{tabular}{cccc}
\cline { 2 - 4 } & \multicolumn{3}{c}{ Edad } \\
\cline { 2 - 4 } & 3 años & 4 años & 5 años \\
\hline Media & 44,26 & 44,35 & 44,84 \\
\hline
\end{tabular}

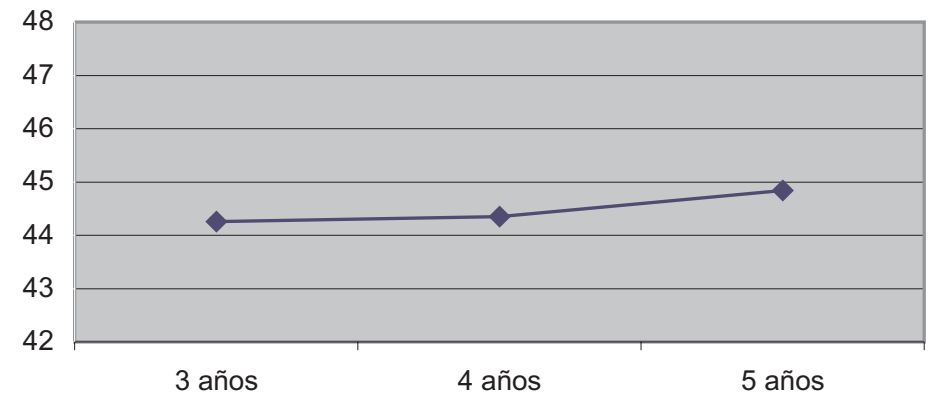


En cuanto a los resultados del examen de reglas alofónicas (Tabla 31), se observó que la media obtenida por los niños de tres años (46.02), fue menor que la media de los niños de cuatro años (47.00), mientras que los niños que cinco años obtuvieron una puntuación media inferior a la obtenida por éstos últimos.

Tabla 31. Perfil del desarrollo de las reglas alofónicas.

\begin{tabular}{cccc}
\cline { 2 - 4 } & \multicolumn{3}{c}{ Edad } \\
\cline { 2 - 4 } & 3 años & 4 años & 5 años \\
\hline Media & 46,02 & 47,00 & 46,89 \\
\hline
\end{tabular}

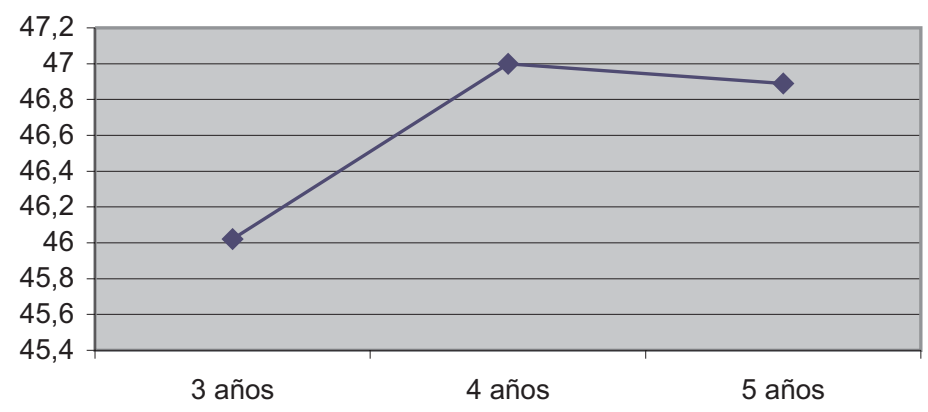

Finalmente, los resultados en cuanto al desarrollo de las reglas de neutralización (Tabla 32), mostraron que el perfil se incrementó de manera progresiva con relación a la edad cronológica.

Tabla 32. Perfil de desarrollo de las reglas de neutralización.

\begin{tabular}{cccc}
\cline { 2 - 4 } & \multicolumn{3}{c}{ Edad } \\
\cline { 2 - 4 } & 3 años & 4 años & 5 años \\
\hline Media & 6,30 & 7,02 & 7,36 \\
\hline
\end{tabular}

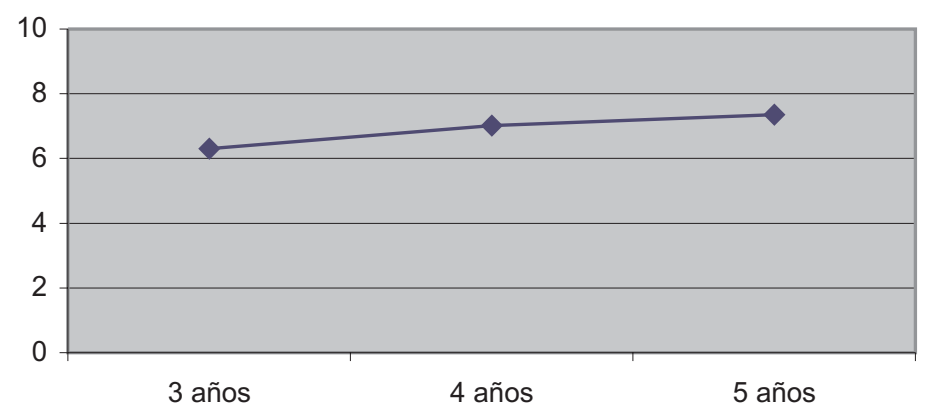




\section{DISCUSIÓN}

El análisis de la conciencia fonológica de rima y aliteración, entendida como la habilidad del niño para reflexionar y manipular la estructura de su sistema fonológico (Acosta; Moreno, 2001), mostró un incremento sostenido y progresivo aunque leve, al considerarse, en forma independiente, las variables edad y nivel socioeconómico, encontrándose que, en el primer caso, dichas diferencias no eran significativas; mientras que en el segundo caso, eran significativamente favorables a los varones.

Lo encontrado es compatible con lo que señalan casi todas las teorías explicativas del desarrollo fonológico, pues se asume que el proceso adquisición fonológica, comienza desde el nacimiento, con la emisión de los primeros sonidos, acentuándose de manera progresiva y gradual hasta aproximadamente la edad de los 4 años, donde la mayoría de los sonidos aparecen discriminados en palabras simples. En el español, el proceso culmina alrededor de los 6 ó 7 años, cuando el niño domina determinadas sílabas y fonemas complejos (Monfort; Juárez, citado en Acosta; León; Ramos 1998: 50).

Por otro lado, los resultados obtenidos en el estudio de la adquisición de reglas alofónicas, refuerza lo descrito por Monfort y Juárez (1996), apreciándose en los niños, un incremento progresivo en el dominio de estas reglas según se incrementó su edad y también, conforme fue mayor el estrato socioeconómico. Sin embargo, al considerarse la variable sexo, ocurrió lo contrario que en conciencia fonológica de rima y aliteración, pues fueron las niñas las que mostraron un mejor rendimiento, siendo el mismo, muy homogéneo, ello coincide con lo afirmado por varios autores en el sentido de que son las niñas las que logran un desarrollo del lenguaje inicial, más rápido que el de los varones (Molina, 1997). En este sentido, es importante recordar que Fey (Acosta; León y Ramos 1998:106) señala que las reglas son «una modificación que hace un niño de una forma adulta, a una forma que es, por razones que no siempre están claras, más manejable para el niño. Esta modificación tiene lugar a nivel subyacente, no periféricamente, de tal modo que los gestos exactos requeridos para una producción, son simplificados de alguna forma», o lo que es lo mismo, es una «operación de sustitución de un fonema por uno de sus alófonos» (Acosta; León, Ramos, 1998:23).

Con respecto a las reglas de neutralización, se encontró un mejor desempeño conforme se incrementó la edad de los examinados; evidenciando las niñas, como en el caso anterior, un mejor rendimiento con respecto a los varones. Los resultados, teniendo en cuenta el nivel socioeconómico, indicaron, al igual que en los casos anteriores, un patrón progresivamente mayor de rendimiento según fue más alto, el nivel socioeconómico.

El estudio de las reglas fonológicas evidenció que las mismas, alcanzaron un mayor desarrollo conforme se incrementó la edad; no ocurriendo ello cuando el análisis se realizó considerando el nivel socioeconómico de procedencia de los niños; sin embargo, en ambos análisis, las diferencias fueron no significativas. Como en la mayor parte de los casos, las niñas mostraron un mejor desempeño con respecto a los varones, aunque las diferencias también fueron no significativas.

Lo encontrado coincide con lo descrito por Treiman y Zukowski en 1991 (Jiménez, 1995), quienes compararon el conocimiento de tres niveles lingüísticos: sílabas, rimas y fonemas en niños de diferentes niveles de preescolar y primer grado. Los hallazgos sugirieron la existencia de una progresión evolutiva que va desde el conocimiento de las sílabas al conocimiento de unidades intrasilábicas (onset-rima), y finalmente, al conocimiento fonético. 
Finalmente, los perfiles mostraron que el rendimiento en conciencia fonológica de rima y aliteración; en reglas fonológicas; en reglas para fonemas con un solo contexto de aparición; y en reglas de neutralización existió un incrementó progresivo de nivel de desempeño, conforme fue mayor la edad cronológica; por el contrario, el perfil de reglas alofónicas fue irregular, no mostrando un patrón secuencial de progreso según la edad.

\section{CONCLUSIONES}

1. La conciencia fonológica de rima y aliteración se incrementó ligera, pero sostenida y progresivamente conforme fue mayor la edad y nivel socioeconómico.

2. El rendimiento en conciencia fonológica de rima y aliteración de los varones es significativamente mejor que el de las mujeres.

3. El dominio de las reglas alofónicas mostró un incremento progresivo conforme se incrementó la edad y el estrato socioeconómico de los escolares.

4. Las niñas alcanzaron un mejor desempeño con respecto a los varones en la adquisición de reglas alofónicas.

5. Conforme se incrementó la edad y el nivel socioeconómico, los varones y mujeres mostraron un mejor dominio de las reglas de neutralización.

6. Las niñas alcanzaron un mejor desempeño con respecto a los varones en el manejo de las reglas de neutralización.

7. Existen diferencias no significativas al comparar el desarrollo de las reglas fonológicas en función a las variables, sexo, edad y nivel socioeconómico.

8. El Test de Desarrollo fonético Fonológico es un instrumento que discrimina adecuadamente los sujetos examinados según su nivel de desempeño, presentando adecuados niveles de validez y confiabilidad.

\section{REFERENCIAS BIBLIOGRÁFICAS}

1. Acosta; Moreno (2001). Dificultades del lenguaje en ambientes educativos. Del retraso al trastorno específico del lenguaje. España: Editorial Masson.

2. Acosta; León; Ramos (1998). Dificultades del habla infantil. Un enfoque clínico. Investigación, teoría y práctica. Málaga: Ediciones ALJIBE.

3. Acosta y otros (1996). La evaluación del lenguaje. Teoría y práctica del proceso de evaluación de la conducta lingüística infantil. Málaga: Ediciones ALJIBE.

4. Arnaiz; Ruiz (2001). La lecto-escritura en la educación infantil: Unidades didácticas y aprendizaje significativo. Málaga: Ediciones ALJIBE.

5. Caravedo, R. (1998). Estudios sobre el español de Lima. Lima: Fondo Editorial de la Pontificia Universidad Católica del Perú.

6. Jiménez, G. (1992). Metaconocimiento fonológico. Madrid: Ed. Síntesis S.A. 
7. Jiménez; Ortiz (1995). Conciencia fonológica y Aprendizaje de la Lectura: Teoría, Evaluación e Intervención. Madrid: Ed. Síntesis S.A.

8. Hernández; Hernández; Baptista (1999). Metodología de la Investigación. México: Mc Graw Hill.

9. Hugo, V. (1991). Comparación de las habilidades psicolingüisticas según el sexo de los alumnos en educación inicial. Tesis para optar el Grado de Bachiller en Psicología. Lima: UEGV.

10. Melgar, M. (1971). Cómo detectar al niño con problemas de habla. $3^{\mathrm{a}}$ ed. México: Edit. Trillas.

11. Molina, S. (1997). El fracaso en el aprendizaje escolar. Dificultades globales de tipo adaptativo. España: Ediciones ALJIBE.

12. Monfort; Juárez (1996). El niño que habla. El lenguaje oral en el preescolar. $6^{\mathrm{a}}$ ed. España: Editorial CEPE. 202 pp

13. Panca, N. (2002). Habilidades metalingüística y rendimiento lector en alumnos de 1er grado de primaria de colegios públicos y privados de San Juan de Lurigancho. Lima: CPAL.

14. Papalia; Wendkos (1997). Psicología del desarrollo. $7^{\text {a }}$ ed. Colombia: Edit. Mac Graw Hill.

15. Ugarriza, N. (1998). Instrumentos para la investigación educacional. Lima: UNMSM. 
$+1$ 